\title{
A novel chimeric DNA vaccine: Enhancement of preventive and therapeutic efficacy of DNA vaccine by fusion of Mucin 1 to a heat shock protein 70 gene
}

\author{
DAE-HAN CHOI ${ }^{1,2 *}$, JONG KYU WOO $^{1-3^{*}}, \mathrm{YUN} \mathrm{CHOI}^{1,2}, \mathrm{HYE}-\mathrm{SOOK} \mathrm{SEO}^{4}$ and CHUL-WOO KIM ${ }^{1-3}$ \\ ${ }^{1}$ Tumor Immunity Medical Research Center; ${ }^{2}$ Cancer Research Institute; ${ }^{3}$ Department of Pathology, \\ College of Medicine, Seoul National University, Seoul 110-799; ${ }^{4}$ Department of Oriental Medicine, \\ Kyung Hee University, Seoul 130-701, Republic of Korea
}

Received March 4, 2011; Accepted June 9, 2011

DOI: $10.3892 / \mathrm{mmr} .2011 .525$

\begin{abstract}
Intensive efforts to improve vaccines against cancer are currently outgoing. Mucin 1 (Muc1) is a tumor-specific antigen that is overexpressed and heavily glycosylated in a variety of adenocarcinomas. In the present study, the efficacy of an anticancer DNA vaccination strategy was demonstrated using Mucl fusion vaccines. To enhance antigen presentation and tumor-suppressive efficacy, a chimeric Mucl vaccine was designed, encoding the transmembrane- and C-terminal domain-deleted Mucl gene ( $\Delta \mathrm{TM})$ fused to the human HSP70 gene. To confirm the expression and secretion of fusion protein, cell culture supernatants were subjected to Western blotting. We found secreted Muc1 $\triangle T M-H S P 0$ fusion protein in the supernatants. These results demonstrate that the Muc1 $\triangle \mathrm{TM}-\mathrm{HSPO}$ construct can be efficiently expressed and secreted from transfected cells. When the chimeric Muc1 vaccine was administered to mice, antigen-specific cellular immune responses were observed. Notably, we observed that antigenspecific lymphocyte proliferation and cytotoxic responses were effectively induced only in the group of mice that had been vaccinated with the chimeric Muc1 vaccine. Concurrent with the Muc1-specific tumor-suppressive effect, the growth of established Muc1-expressing B16 mouse melanoma cells was also significantly inhibited by vaccination with the chimeric Muc1 vaccine. The growth of B16 mouse melanoma cells expressing human Muc1 in C57BL/6 mice was effectively suppressed by the Muc1-HSP70 chimeric DNA vaccine. Our
\end{abstract}

Correspondence to: Dr Chul-Woo Kim, Department of Pathology, College of Medicine, Seoul National University, 28 Yeongeon-dong, Jongno-gu, Seoul 110-799, Republic of Korea

E-mail: cwkim@snu.ac.kr

*Contributed equally

Key words: Muc1, HSP70, chimeric DNA vaccine, cancer, immunotherapy results reveal that the antitumor efficacy of the chimeric DNA vaccine was improved by the presence of HSP/70.

\section{Introduction}

DNA vaccination is an attractive method for inducing an anticancer immune response. DNA vaccines are easily prepared and have low immunogenicity in that they may be injected repeatedly and are highly stable compared to viral-based or other vaccine forms $(1,2)$. Their effectiveness for use in rapid screening for antigens and the design of specific types of expression constructs has made the study of DNA vaccines valuable for developing immunotherapies for cancer (2). The goal of DNA vaccination is the development of effective immunization strategies against previously established tumors. Strategies for improving DNA vaccines, such as, fusion of adjuvant proteins in construction or use of immune modulators for improvement of immunogenicity, or the use of next-generation delivery methods, are under intensive investigation $(3,4)$.

Mucin 1 (Muc1) is a well-studied tumor antigen that is overexpressed in a number of human adenocarcinomas $(5,6)$. Mucl is translated as a single precursor polypeptide and undergoes proteolytic cleavage into two subunits that are composed of an extracellular unit associated with the transmembrane and cytoplasmic tail form (7). Mucl is potentially an ideal antigen for immune-based therapy due to its changes in intracellular distribution, which occur on the apical cell surface in normal glandular epithelia, are widespread on the surface of cancer cells.

Heat shock proteins (HSPs), including HSP70, HSP90, GP96 and calreticulin, are molecular chaperones that are highly effective stimulators in innate and adaptive immune responses (8). In particular, HSP70 generates cross-priming events and is a highly effective molecule involved in the promotion of immune responses via interaction with several surface receptors on antigen-presenting cells (APCs) and antigen-specific $\mathrm{CD}^{+}$cytotoxic $\mathrm{T}$ lymphocyte (CTL) responses (9-12).

In the present study, the intramuscular administration of a chimeric DNA construct, comprising the transmembrane and C-terminal domain-deleted Mucl gene $(\Delta \mathrm{TM})$ as an antigen 
and the human HSP70 gene as an adjuvant, was investigated. A leader sequence of tyrosinase was also added to guide the fusion protein into the endoplasmic reticulum for secretion. Thus, this secreted fusion protein was capable of facilitating antigen presentation in APCs via HSP70-receptor mediated interaction. The results of this study showed that the secreted fusion protein from Muc1 $\Delta$ TM-HSP70 DNA vaccination was sufficient to induce antigen-specific immune responses in C57BL/6 mice, and prevented tumor progression prophylactically in preventive and therapeutic mouse models.

\section{Materials and methods}

DNA constructs and cell clones. The human mucin 1 gene, Muc1, (GenBank: J05582) was cloned into the pcDNA3 vector (Invitrogen, Carlsbad, CA, USA), as previously described (13). Cloning of full-length HSP70 cDNA and human tyrosinase leader peptide were performed using primers HSP70 (5'-TTT GGA TCC ATG GCC AAA GCC GCG GCG AT-3' and 5'-TTT CTC GAG GGC CCC TAA TCC ACC TCC TCA ATG G-3') and human tyrosinase leader peptide (5'-TTT AAG CTT ATG CTC CTA GCT GTT TTG TAC-3' and 5'-TTT GGA TCC GGC TCT AGG GGA AAT GGC CAG C-3'). In brief, the human Mucl gene, transmembrane- and C-terminal $\triangle \mathrm{TM}$, secretory human HSP70 gene (sHSP70) or secretory $\Delta$ TM-HSP70 fusion gene $(\mathrm{MH})$ were synthesized by PCR and placed into the pcDNA3 vector.

A human Muc1-expressing B16/Mucl cell line was generated from $\mathrm{B} 16-\mathrm{F} 1$ mouse melanoma cells following infection with human Muc1-encoding retrovirus pLXIN, as previously described (14).

Transfection and Western blotting. HEK-293 cells were transfected with DNA vaccine constructs (Muc1, $\Delta \mathrm{TM}$, sHSP70 and $\mathrm{MH}$ ) by Lipofectamine 2000 reagent (Invitrogen). After 36 h, cell lysates were extracted with modified-RIPA buffer (50 mMTris$\mathrm{HCl}, 150 \mathrm{mM} \mathrm{NaCl}, 1 \% \mathrm{NP}-40,0.25 \% \mathrm{Na}$-deoxycholate and $1 \mathrm{mM}$ EDTA) containing a protease inhibitor mixture (Roche, San Francisco, CA, USA). Conditioned medium was prepared from HEK-293 cells. In brief, HEK-293 cells were transfected with DNA vaccine constructs. Cells were then cultured until $\sim 90 \%$ confluence was achieved, and the medium was exchanged with serum-free medium for a further $12 \mathrm{~h}$. Serumfree conditioned media were collected and concentrated by Centriplus (Millipore, Billerica, MA, USA). Total cell lysates (50 $\mu \mathrm{g} /$ well) and concentrated conditioned media (20 $\mu \mathrm{l} /$ well) were separated on a 6 or $10 \%$ SDS-PAGE gel. The separated proteins were subsequently transferred to a PVDF membrane (Millipore). The membranes were labeled with primary antibody against Muc1 (Biomeda, Burlingame, CA, USA) and HSP70 (Stressgen, Plymouth Meeting, PA, USA).

In vitro cellular immune response. Splenocytes were harvested from vaccinated mice 7 days after the 2 nd vaccination and cells were labeled with $10 \mu \mathrm{M}$ carboxyl fluorescein diacetate succinimidyl ester (CFSE; Molecular Probes, Urbana, IL, USA) for $10 \mathrm{~min}$ at $37^{\circ} \mathrm{C}$, following which they were cultured in the presence of $5 \mu \mathrm{g} / \mathrm{ml}$ Mucl peptide for 5 days. Following stimulation, splenocytes were collected and incubated with biotin-conjugated anti-TCR- $\beta$ (H57-597,
BD Pharmingen, San Diego, CA, USA), then stained with Avidin-PE (BD Pharmingen). Proliferations were analyzed by flow cytometry. The Mucl peptide used contained the sequence PDTRPAPGSTAPPAHGVTSAPDTRPAPGST, as previously described (15). The peptide was synthesized and obtained from Peptron (Daejeon, Republic of Korea).

Enzyme-linked immunospot assays. The number of IFN- $\gamma$ producing cells was also determined by an enzyme-linked immunospot assay (ELISPOT). The 96-well multi-screen HTS plates (Millipore) were coated overnight with $5 \mu \mathrm{g} / \mathrm{ml}$ of antiIFN- $\gamma$ antibody (R4-6A2, BD Pharmingen). Freshly collected splenocytes were plated on an HTS plate and incubated with the Muc1 peptide $(10 \mu \mathrm{g} / \mathrm{ml})$ for $24 \mathrm{~h}$. The biotinconjugated anti-IFN- $\gamma$ antibody (XMG1.2, BD Pharmingen) was added to the plate. Avidin-HRP (Amersham Biosciences, Piscataway, NJ, USA) and AEC (BD Pharmingen) were used to detect IFN- $\gamma$ positive spots. The plates were then counted with an Elispot 04 SR (Advanced Imaging Devices $\mathrm{GmbH}$, Strassberg, Germany).

Intracellular IFN- $\gamma$ staining. Intracellular cytokine staining was performed using a BD Cytofix-Cytoperm kit with BD Golgistop (BD Pharmingen) according to the manufacturer's protocol. Fresh splenocytes were prepared from immunized mice and stimulated in vitro for $24 \mathrm{~h}$ with $10 \mu \mathrm{g} / \mathrm{ml} \mathrm{Mucl}$ peptide. Splenocytes were treated with Golgistop and then stained with FITC-conjugated $\mathrm{mAb}$ against CD8 $\alpha$ (BD Phamingen) and intracellular cytokine was stained with PE-conjugated mAb IFN- $\gamma$ (BD Pharmingen).

Antigen-specific cytotoxic assay. The CytoTox 96 non-radioactive cytotoxicity assay (Promega, Sunnyvale, CA, USA) was used according to the manufacturer's protocol for the determination of cytotoxic activity. Splenocytes were collected from mice administered with the DNA vaccines, and then cells were stimulated with recombinant human interleukin-2 $(50 \mathrm{U} / \mathrm{ml})$ and $10 \mu \mathrm{g} / \mathrm{ml}$ Mucl peptide. Human Muc1 expressing B16/F1 murine melanoma and parental B16/F1 cell lines were used as target cells. Radiation-treated target cells were dispensed into the wells of U-bottomed 96-well plates. A number of the activated splenocytes were added in triplicate. Plates were then incubated for $5 \mathrm{~h}$ in a humidified $5 \% \mathrm{CO}_{2}$ chamber at $37^{\circ} \mathrm{C}$. Supernatants $(50 \mu \mathrm{l})$ were transferred to fresh 96 -well flatbottomed plates, and an equal volume of reconstituted substrate mix was added to each well. The plates were then incubated in the dark at room temperature for $30 \mathrm{~min}$ and absorbance values were measured at $492 \mathrm{~nm}$. Cell death percentages for each effector cell to target cell ratio were calculated using the formula: [A (experimental) - A (effector spontaneous) - A (target spontaneous)] x 100/[A (target maximum) - A (target spontaneous)]. These assays were performed at 1/50, 1/25, 1/10 and $1 / 5$ effector $(\mathrm{E}) /$ target $(\mathrm{T})$ ratios.

In vivo tumor suppression assay. Specific pathogen-free (SPF) 6-week-old female C57BL/6 mice were purchased from SLC, Inc. (Shizuoka, Japan). For prevention models, C57BL/6 mice were vaccinated twice (2-week interval). B16/Muc1 cells $\left(2 \times 10^{5}\right)$ were injected subcutaneously in the right flank. Tumors were measured twice a week with digital calipers 
A

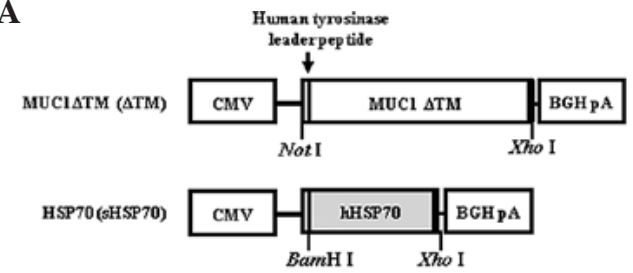

$\triangle \mathrm{TM} \cdot \mathrm{HSP} 70$ (MH)

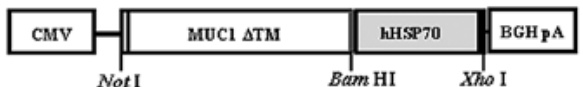

B

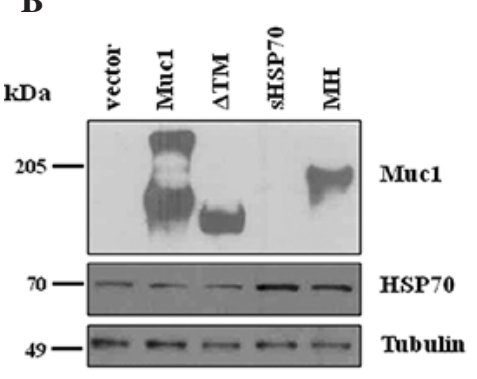

C

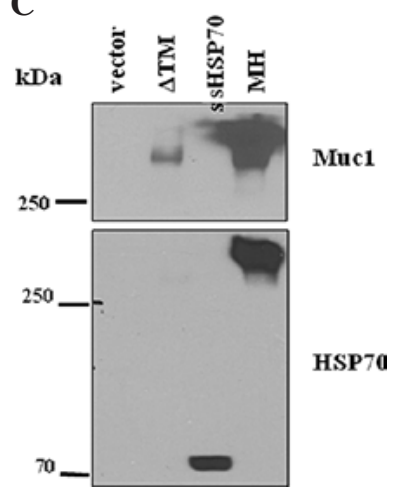

Figure 1. Characterization of Muc1-HSP70 (MH) expressing constructs. (A) Schematic representation of the DNA constructs. The secretory transmembrane- and C-terminal domain-deleted Mucl gene $(\Delta \mathrm{TM})$, secretory human HSP70 gene (sHSP70) or secretory $\Delta \mathrm{TM}-\mathrm{HSP} 70$ fusion gene $(\mathrm{MH})$ were placed under control of the CMV-promoter in the pcDNA3 vector. Not I, Bam HI and Xho I are the employed cloning sites. (B) Total cell lysates from transfected 293 cells were resolved by SDS-PAGE (6 or 10\%) and Western blotting was performed. (C) Culture mediums from transfected cells were concentrated 10 -fold. Proteins secreted into the media were analyzed with anti-Muc1 and anti- HSP70 antibodies.

1 week after the second vaccination. To test the efficacy of the therapeutic DNA vaccination, C57BL/6 mice were challenged with $5 \times 10^{4} \mathrm{~B} 16 / \mathrm{Muc1}$ cells in the right flank. On days 3, 10 and 17 following tumor cell inoculation, mice were vaccinated intramuscularly with $100 \mu \mathrm{g}$ of DNA vaccine and then monitored twice a week for tumor growth. Tumor volume was calculated according to the formula: length $\mathrm{x}$ width ${ }^{2} \mathrm{x} 0.523$.

Statistical analysis. Data were expressed as the mean \pm SD and were representative of at least 2 separate experiments. Statistical significance was determined using Student's t-test. $\mathrm{P}<0.05$ was considered to be statistically significant.

\section{Results}

Generation and characterization of the secreted $M H$ construct. The DNA construct, MH (Muc1 $\triangle \mathrm{TM}-\mathrm{HSP} 70$ ), was generated by fusing the extracellular domain of human Muc1 and human HSP70, and was controlled by the CMV promoter. For the secretion of $\mathrm{MH}$ fusion protein, the tyrosinase leader peptide was inserted into the $\mathrm{N}$-terminal region of the Mucl gene (Fig. 1A). To determine MH fusion protein expression levels, DNA constructs were transiently transfected into HEK-293, and cell lysates were subjected to Western blotting.
The molecular mass of $\mathrm{MH}$ was shifted compared to that of Muc1 $\triangle \mathrm{TM}$ and non-glycosylated Muc1 in the transfected cells (Fig. 1B). To confirm the secretion of MH, cell culture supernatants were subjected to Western blotting. Secreted MH was observed in the supernatants (Fig. 1C). Taken together, the results show that the MH construct may be efficiently expressed and secreted from transfected cells.

MH vaccination induced Mucl-specific T-cell response. To determine whether the $\mathrm{MH}$ vaccine was capable of inducing specific cellular immune responses against Muc1, C57BL/6 mice were vaccinated with $100 \mu \mathrm{g}$ of DNA constructs, which encoded each of sHSP70, $\Delta \mathrm{TM}$ and MH. We first determined whether splenocytes from vaccinated mice would respond to antigenic stimulation. Proliferation of antigen-specific T cells was analyzed using a CFSE-based cell division assay and flow cytometry. Splenocytes from mice vaccinated with $\Delta \mathrm{TM}$ and $\mathrm{MH}$ constructs revealed a strong proliferation response compared to the other groups (Fig. 2A). In particular, the MH-vaccinated group of mice $(65.5 \pm 12.3 \%)$ showed a higher cell proliferation response compared to the $\Delta \mathrm{TM}$-vaccinated group $(41.8 \pm 11.5 \%)$. To further characterize the immune response, ELISPOT and intracellular cytokine staining assays were performed. The frequency of IFN- $\gamma$-secreting splenic T cells was assessed by an ELISPOT assay. As shown in Fig. 2B, mice vaccinated with $\mathrm{MH}$ had significantly more IFN- $\gamma$ secreting $\mathrm{T}$ cells in the spleen when compared to the remaining groups $(\mathrm{P}<0.01)$. In addition, the Muc1-specific $\mathrm{CD} 8^{+} \mathrm{T}$ cells were characterized by intracellular IFN- $\gamma$ staining. As shown in Fig. $2 \mathrm{C}$, the $\mathrm{MH}$ vaccination group generated a significantly higher number of Muc1-specific $\mathrm{CD} 8^{+} / \mathrm{IFN}-\gamma^{+}$double-positive T cells as compared to the other groups $(\mathrm{P}<0.01)$. Thus, our data indicate that vaccination with the $\mathrm{MH}$ construct was capable of inducing stronger Muc1-specific cellular immune responses and $\mathrm{CD}^{+} \mathrm{T}$-cell immune responses.

Vaccination with MH generates Mucl-specific cytotoxic response. We evaluated whether $\mathrm{MH}$ vaccination was capable of enhancing the efficacy of Muc1-specific cytotoxic activity against the B16/Muc1 melanoma cell lines. The syngenistic, C57BL/6 melanoma cell line, B16F1, expressed human Muc1. Cytotoxic activity was only observed when mice were immunized with the MH vaccine $(\mathrm{P}<0.01$ as compared to the other groups), confirming that immunization elicited CTLs specifically against the Muc1 antigen. Mice vaccinated with vector sHSP70 and $\triangle \mathrm{TM}$ exhibited less cytotoxic activity against B16/Muc1 (Fig. 3). As shown in Fig. 3, Muc1-specific CTL activity was exhibited when B16/Mucl were target cells. However, with B16/neo cells, Muc1-specific CTL activity was not observed in splenocytes from $\mathrm{MH}$-vaccinated mice. The results indicate that $\mathrm{MH}$ vaccination strongly induced a cytotoxic cellular response against Muc1 as a tumor antigen.

Inhibition of tumor growth in C57BL/6 mice by immunization with $M H D N A$ vaccine. In order to determine whether MH DNA vaccination generated tumor suppressive activity, the DNA vaccine was tested for preventive and therapeutic effect against B16/Muc1 in C57BL/6 mice. In the prevention assay (Fig. 4A), strong suppression of B16/Muc1 growth was observed in C57BL/6 mice that received the MH DNA vaccine 
A
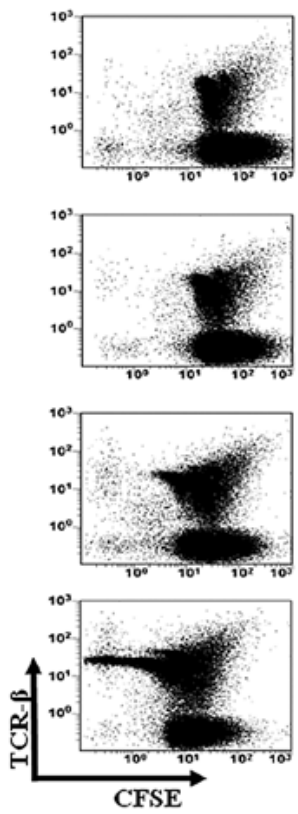

TCR- $\beta^{+}$cells
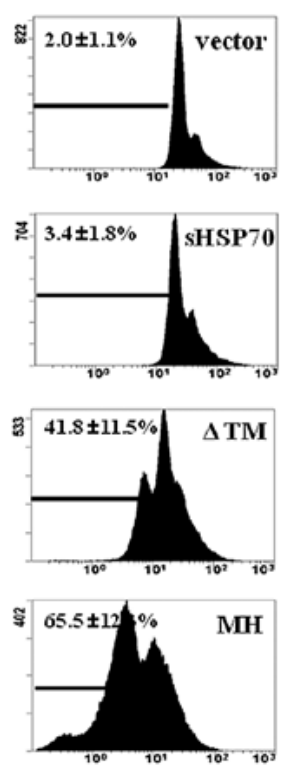

B

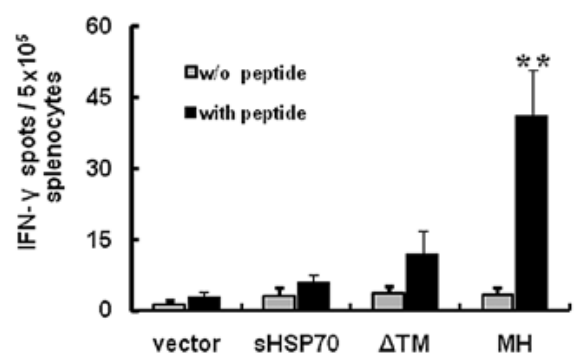

C

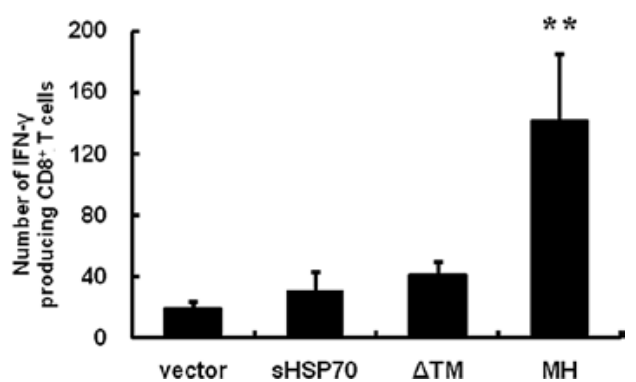

Figure 2. Muc1-specific cellular immune response was induced by MH vaccination. Splenocytes were collected from vaccinated mice spleen 7 days after vaccination (n=3 mice per group). (A) Vaccinated mice splenocytes were labeled with $10 \mu \mathrm{M}$ CFSE and then cultured with $5 \mu \mathrm{g} / \mathrm{ml}$ Mucl peptide for 5 days. Cultured splenocytes were incubated with biotin-conjugated anti-TCR- $\beta$ and then stained with avidin-PE. Cell proliferation rates were determined by flow cytometry. Cells were gated on the TCR- $\beta^{+}$population. (B) Mucl peptide-stimulated splenocytes from vaccinated mice were examined for IFN- $\gamma$-producing cells by ELISPOT. Splenocytes were stimulated with Muc1 peptide $(10 \mu \mathrm{g} / \mathrm{ml})$ for $24 \mathrm{~h}$ to assay. Non-stimulated cells were used as a negative control and ConA ( $1 \mu \mathrm{g} / \mathrm{ml})$ was used as a positive control (data not shown). A representative example of 2 different experiments (n=3 mice per group) is shown. (D) Muc1-specific CD8 ${ }^{+} \mathrm{T}$ cells were evaluated by IFN- $\gamma^{+}$staining. Splenocytes were stimulated with Muc1 peptide (10 $\left.\mu \mathrm{g} / \mathrm{ml}\right)$ and then staining for CD8 and intracellular IFN $-\gamma$ was performed. Data represent one of two independent experiments with different mice. Error bars are the means \pm SD. ${ }^{* *} \mathrm{P}<0.01$.

( $\mathrm{P}<0.01$ as compared to all other groups). Of 7 mice in the $\mathrm{MH}$-vaccinated group, 4 remained tumor-free (Fig. 4B). Mice in all other treatment groups developed tumors, but with a lower incidence and lower tumor weight compared to mice treated with vehicle. Our data indicate that vaccination with $\mathrm{MH}$ induces Mucl-specific immunity, which was sufficient to prevent and delay the growth of Muc1-expressing tumors in vivo. For the therapeutic study model, the tumor-bearing mice ( $n=8$ per group) were vaccinated with $100 \mu \mathrm{g}$ of DNA vaccine constructs and boosted twice. In the $\mathrm{MH}$ vaccination group, 6 mice developed tumor masses (data not shown). However, these tumor masses were small as compared to the other immunized groups $(\mathrm{P}<0.05$; Fig. $4 \mathrm{C})$. Mice in the sHSP70 and $\Delta \mathrm{TM}$ groups developed larger tumor masses after tumor inoculation compared to the $\mathrm{MH}$ group. The treatment schedules were started according to the experimental design shown in Fig. 4D with the vehicle, sHSP70, $\triangle \mathrm{TM}$ and $\mathrm{MH}$. Thus, the results indicate that Muc1-HSP70 fusion DNA vaccination exhibited, not only preventive, but also therapeutic effects against in vivo tumor growth.

\section{Discussion}

Successful approaches for tumor immunotherapy should overcome the immune system's tolerance of self-tumor antigens. Improvements in vaccine design are required for efficient cancer immunotherapy (16-19). In the present study, a cancer DNA vaccine was developed designed to enhance the efficacy of cancer therapy. Chimeric DNA vaccine designs were investigated, in which the antigen of interest was expressed as an in-frame fusion with HSP70 that impacted antigen processing or presentation.

Tumor-associated Mucl is known to be recognized by the immune system in cancer patients. The tandem repeat domain of the Mucl core protein and signal sequences contain epitopes that are recognized by CTLs in both mice and humans $(5-6,20)$. Compared to other Muc1-derived DNA vaccines, adjuvants have been used to enhance the potency of Mucl DNA vaccines such as Flt-3 ligand (15), GM-CSF (21), and IL-18 (22) injection and antigen fused form expression plasmids. Two specific methods were used in the development of our MH vaccine. First, the leader sequence was inserted to the N-terminal region of the fusion protein, which was capable of enhancing the expression of the secreted form of epitopes in muscle cells, thereby inducing more specific CTL activity. Second, the vaccine contained an HSP70 gene, which made it much more specific for APC targeting. HSP70 was selected as the targeting molecule for secreted antigen due to its physiological role as a carrier molecule of peptides from necrotic cells into the MHC Class I cross-presentation pathway of APCs. Alternatively, the intratumoral injection of plasmid predominantly led to the transfection of mycocytes. Mycocytes lack an expression of MHC II and costimulatory molecules, and thus would not be expected to prime T lymphocytes directly. Therefore, efforts have been made to enhance the potency of DNA vaccination by facilitating transport of these antigens into the MHC Class I 
A

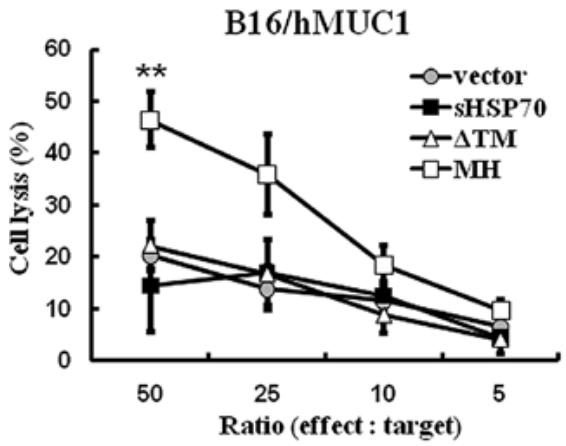

B

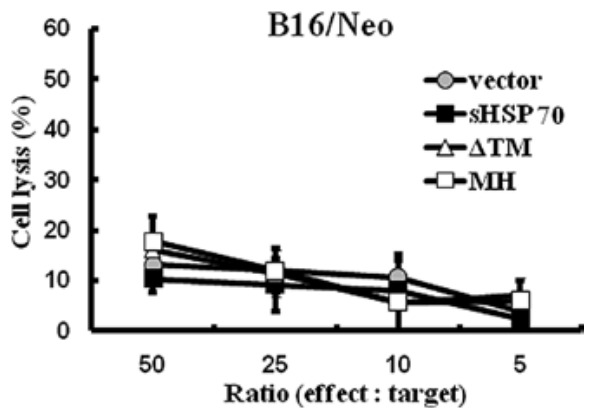

Figure 3. MH DNA vaccination enhanced cytotoxic efficacy of splenocytes. Mice ( $\mathrm{n}=3$ per group) were immunized through intramuscular injection with PBS, sHSP70, $\Delta \mathrm{TM}$, or MH DNA plasmid (100 $\mu \mathrm{g}$ per mouse) twice with a 2-week interval. (A) B16/Muc1 or (B) B16F1/Neo cells were used as target cells. Different effector-to-target cell ratios (50:1, 25:1, 10:1, and 5:1) were mixed for $5 \mathrm{~h}$. Specific lysis of target cells was measured by lactate dehydrogenase release and calculated using the formula as recommended for the CytoTox 96 assay kit (Promega). Data represent one of two independent experiments with different mice. Error bars are the means $\pm \mathrm{SD}$. ${ }^{* *} \mathrm{P}<0.01$. antigen presentation pathway of APCs. A leader peptide was exploited to re-route the antigen-HSP70 fusion protein into the endoplasmic reticulum for secretion. The secreted fusion protein is targeted and captured by APCs via interaction of HSP70 with its cell surface receptors, bringing the antigen into the MHC Class I cross-presentation pathway and delivering a maturation signal to APCs (23-25). These characteristics enhance the priming of antigen-specific $\mathrm{T}$ cells and lead to a strong antitumor effect.

Our observations indicated that intramuscular injections of MH vaccine suppressed the development of human Muc1expressing syngenic mouse melanoma cancer in C57BL/6 mice (Fig. 4). Additionally, our therapeutic vaccine model revealed inhibitory effects of the vaccine on tumor growth. The Muc1specific cytotoxicity assay revealed that splenocytes from MH-vaccinated mice enhanced the specific cytotoxic activity among 4 DNA vaccine groups. Moreover, this vaccination strategy was noted to generate a Muc1-specific cellular immune response (Fig. 3). Mouse melanoma cells expressing human Muc1 were effectively identified and lysed by splenocytes from MH-vaccinated mice with as compared to mock control cells (B16/neo). Thus, DNA-based vaccines using HSP70 could potentially play a crucial role in cancer immune therapy.

In the present study, MH cancer vaccine was shown to enhance preventive and therapeutic antitumor efficacy, although the mechanism for this efficacy of the MH constructs is not clear. If the MH vaccine generates HSP-specific cellular immune responses, these may contribute to the generation and expansion of Muc1-specific immune responses. It has been

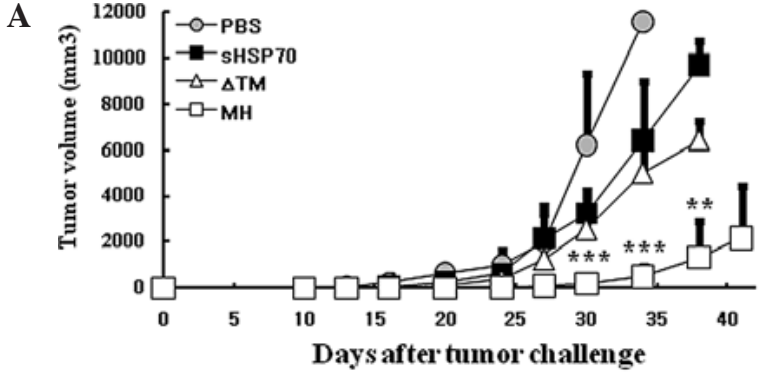

C

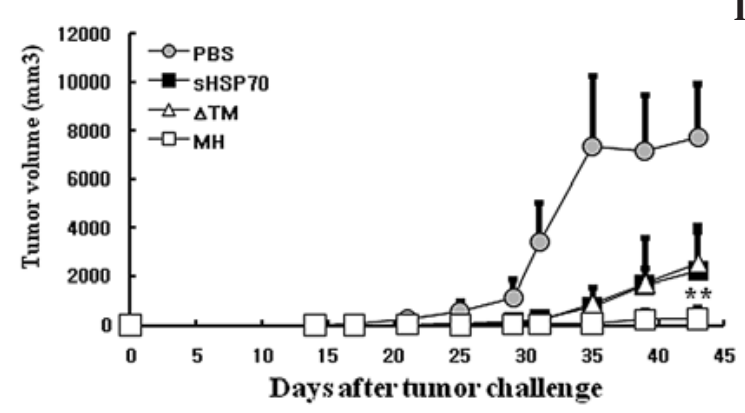

B

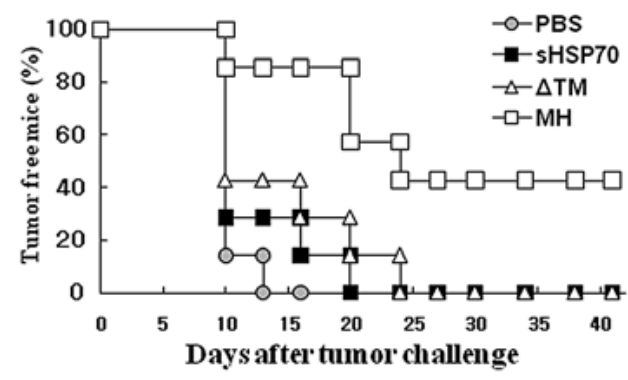

D

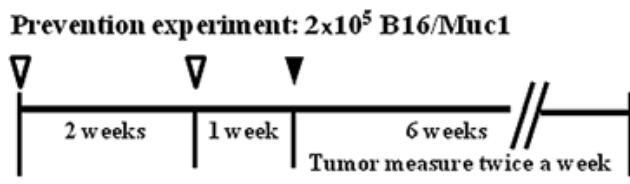

Therap eutic experiment: $5 \times 10^{4}$ B16/Muc1

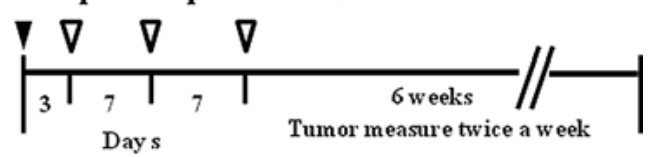

Tumor challenge $\nabla$ vaccination

Figure 4. In vivo tumor suppressive efficacy was enhanced by MH DNA vaccination. In prevention experiments, mice ( $\mathrm{n}=7-8$ per group) were immunized with vehicle, sHSP70, $\Delta \mathrm{TM}$ or MH DNA plasmid (100 $\mu \mathrm{g}$ per mouse). Mice were challenged subcutaneously with $2 \times 10^{5} \mathrm{~B} 16 / \mathrm{Muc1}$ tumor cells after 21 days. (A) Mice bearing B16/Muc1 melanoma tumors were monitored for 6 weeks. (B) Data are presented as the percentage of tumor-free mice in the prevention experiment. (C) Tumor volume demonstrating therapeutic effects of the MH vaccination. C57BL/6 mice (n=7-8 per group) were challenged subcutaneously with $5 \times 10^{4} \mathrm{~B} 16 / \mathrm{MUC} 1$ tumor cells (day 0 ). The DNA vaccines (100 $\mu \mathrm{g}$ per mouse) were administered intramuscularly on day 3,10 and 17 . At each time point, tumor sizes of each mouse from a given group were measured and compared statistically with those of the other group. Data represent one of two independent experiments with different mice. (D) In vivo experiment schedule. Tumors were established in C57BL/6 mice with B16/Muc1 mouse melanoma cells for tumor prevention and therapeutic experiments. Error bars are the means $\pm \mathrm{SD} .{ }^{* * *} \mathrm{P}<0.001$ and ${ }^{* *} \mathrm{P}<0.01$. 
suggested that a high precursor frequency of HSP70-specific $\mathrm{T}$ cells exists due to the continual exposure of the immune system to HSP70 from necrotic or inflammatory regions of the human body (25). An alternative hypothesis is that the HSP70 enhances the potency of DNA vaccination by facilitating transport of these antigens into the MHC Class I antigen presentation pathway of APCs (24).

In conclusion, our data show that fusion of a secreted tumor antigen to HSP70 generates a significantly improved DNA vaccine as compared to vaccines that encode antigen only. This concept should apply equally well to antigens other than the Mucl tumor-associated antigen, and to other types of tumor vaccine. More studies are currently underway to determine the mechanisms for enhanced efficacy of MH constructs.

\section{Acknowledgements}

This study was supported in part by grants from The Cancer Research Center of the Korean Science and Engineering Foundation (KOSEF) through the Tumor Immunity Medical Research Center at the College of Medicine, Seoul National University, Republic of Korea.

\section{References}

1. Yannelli JR and Wroblewski JM: On the road to a tumor cell vaccine: 20 years of cellular immunotherapy. Vaccine 23: 97-113, 2004.

2. Fioretti D, Iurescia S, Fazio VM and Rinaldi M: DNA vaccines: developing new strategies against cancer. J Biomed Biotechnol: Mar 28, 2010 (E-pub ahead of print).

3. Ferrone CR, Perales MA, Goldberg SM, et al: Adjuvanticity of plasmid DNA encoding cytokines fused to immunoglobulin Fc domains. Clin Cancer Res 12: 5511-5519, 2006.

4. Ruffini PA, Grodeland G, Fredriksen AB and Bogen B: Human chemokine MIP1alpha increases efficiency of targeted DNA fusion vaccines. Vaccine: Nov 4, 2010 (E-pub ahead of print).

5. Gendler S, Taylor-Papadimitriou J, Duhig T, Rothbard J and Burchell J: A highly immunogenic region of a human polymorphic epithelial mucin expressed by carcinomas is made up of tandem repeats. J Biol Chem 263: 12820-12823, 1988.

6. Vlad AM, Kettel JC, Alajez NM, Carlos CA and Finn OJ: MUC1 immunobiology: from discovery to clinical applications. Adv Immunol 82: 249-293, 2004.

7. Parry S, Silverman HS, McDermottK, Willis A, Hollingsworth MA and Harris A: Identification of MUC1 proteolytic cleavage sites in vivo. Biochem Biophys Res Commun 283: 715-720, 2001.

8. Srivastava P: Roles of heat-shock proteins in innate and adaptive immunity. Nat Rev Immunol 2: 185-194, 2002.
9. Chen $\mathrm{CH}$, Wang TL, Hung CF, et al: Enhancement of DNA vaccine potency by linkage of antigen gene to an HSP70 gene. Cancer Res 60: 1035-1042, 2000.

10. Cho BK, Palliser D, Guillen E, et al: A proposed mechanism for the induction of cytotoxic T lymphocyte production by heat shock fusion proteins. Immunity 12: 263-272, 2000.

11. Castelli C, Ciupitu AM, Rini F, et al: Human heat shock protein 70 peptide complexes specifically activate antimelanoma T cells. Cancer Res 61: 222-227, 2001.

12. Binder RJ and Srivastava PK: Peptides chaperoned by heat-shock proteins are a necessary and sufficient source of antigen in the cross-priming of CD8 ${ }^{+}$T cells. Nat Immunol 6: 593-599, 2005.

13. Jeon YH, Choi Y, Yoon SO, Kim CW and Chung JK: Synergistic tumoricidal effect of combined hMUC1 vaccination and hNIS radioiodine gene therapy. Mol Cancer Ther 7: 2252-2260, 2008.

14. Cho JA, Yeo DJ, Son HY, et al: Exosomes: a new delivery system for tumor antigens in cancer immunotherapy. Int J Cancer 114: 613-622, 2005

15. Fong CL, Mok CL and Hui KM: Intramuscular immunization with plasmid coexpressing tumour antigen and Flt-3L results in potent tumour regression. Gene Ther 13: 245-256, 2006.

16. Fredriksen AB, Sandlie I and Bogen B: DNA vaccines increase immunogenicity of idiotypic tumor antigen by targeting novel fusion proteins to antigen-presenting cells. Mol Ther 13: 776-785, 2006.

17. Schjetne KW, Fredriksen AB and Bogen B: Delivery of antigen to CD40 induces protective immune responses against tumors. J Immunol 178: 4169-4176, 2007.

18. Fredriksen AB and Bogen B: Chemokine-idiotype fusion DNA vaccines are potentiated by bivalency and xenogeneic sequences. Blood 110: 1797-1805, 2007.

19. Nchinda G, Kuroiwa J, Oks M, et al: The efficacy of DNA vaccination is enhanced in mice by targeting the encoded protein to dendritic cells. J Clin Invest 118: 1427-1436, 2008.

20. Heukamp LC, van Hall T, Ossendorp F, et al: Effective immunotherapy of cancer in MUC1-transgenic mice using clonal cytotoxic T lymphocytes directed against an immunodominant MUC1 epitope. J Immunother 25: 46-56, 2002.

21. Chu Y, Xia M, Lin Y, et al: Th2-dominated antitumor immunity induced by DNA immunization with the genes coding for a basal core peptide PDTRP and GM-CSF. Cancer Gene Ther 13: 510-519, 2006.

22. Snyder LA, Goletz TJ, Gunn GR, et al: A MUC1/IL-18 DNA vaccine induces antitumor immunity and increased survival in MUC1 transgenic mice. Vaccine 24: 3340-3352, 2006.

23. Hauser H and Chen SY: Augmentation of DNA vaccine potency through secretory heat shock protein-mediated antigen targeting. Methods 31: 225-231, 2003.

24. Hauser H, Shen L, Gu QL, Krueger S and Chen SY: Secretory heat-shock protein as a dendritic cell-targeting molecule: a new strategy to enhance the potency of genetic vaccines. Gene Ther 11: 924-932, 2004.

25. Millar DG, Garza KM, Odermatt B, et al: Hsp70 promotes antigen-presenting cell function and converts T-cell tolerance to autoimmunity in vivo. Nat Med 9: 1469-1476, 2003. 